Gut and Liver, Vol. 9, No. 3, May 2015, pp. 405-410

\title{
Usefulness of the Controlled Attenuation Parameter for Detecting Liver Steatosis in Health Checkup Examinees
}

\author{
Ja Kyung Kim ${ }^{*}{ }^{\dagger}$, Kwan Sik Lee*, ${ }^{\dagger}$, Jung Ran Choi*, Hyun Jung Chung*, Da Hyun Jung*, Kyung Ah Lee**, ${ }^{\dagger}$, and Jung II \\ Lee $^{*,+}$ \\ ${ }^{*}$ Department of Internal Medicine, Gangnam Severance Hospital, Yonsei University College of Medicine, and ${ }^{\dagger}$ Liver Cirrhosis Clinical Research \\ Center, Seoul, Korea
}

Background/Aims: The controlled attenuation parameter (CAP) implemented in FibroScan ${ }^{\circledR}$ is reported to be a noninvasive means of detecting steatosis ( $>10 \%$ steatosis). We aimed to evaluate the usefulness of CAP in detecting steatosis among health checkup examinees and to assess its correlation with ultrasonography (US). Methods: Consecutive CAP results were retrospectively collected. A total of 280 subjects were included. Results: Fatty liver was detected in 119 subjects (42.5\%) by US, whereas it was detected in 160 subjects (57.1\%) by the CAP. The numbers of subjects with S0:S1:S2:S3 steatosis according to the CAP value were 120:59:58:43, respectively. The mean CAP values were 203.34 $\pm 28.39 \mathrm{~dB} / \mathrm{m}$ for S0, $248.83 \pm 6.14 \mathrm{~dB} / \mathrm{m}$ for $\mathrm{S} 1$, $274.33 \pm 8.53 \mathrm{~dB} / \mathrm{m}$ for $\mathrm{S} 2$, and $322.35 \pm 22.20 \mathrm{~dB} / \mathrm{m}$ for S3. CAP values were correlated with body weight $(r=0.404$, $p<0.001)$, body mass index $(r=0.445, p<0.001)$, and the fatty liver grade by US ( $r=0.472, p<0.001)$. Among the 161 subjects with normal US findings, steatosis was detected in 65 subjects (40.4\%) using the CAP. Conclusions: The CAP seems to be useful for detecting very low-grade hepatic steatosis in health checkup examinees. Its role in predicting subjects with a risk of metabolic derangement needs to be evaluated. (Gut Liver 2015;9:405-410)

Key Words: Fatty liver; Controlled attenuation parameter; UItrasonography; Early diagnosis; Metabolic syndrome

\section{INTRODUCTION}

Nonalcoholic fatty liver disease (NAFLD) is a component of the metabolic syndrome which consists of insulin resistance, visceral obesity, dyslipidemia, hypertension and diabetes mel- litus. ${ }^{1}$ It is the most prevalent chronic liver disease worldwide, ranging from simple steatosis to steatohepatitis, where the presence and the severity of inflammation and fibrosis are important prognostic factors pertaining liver associated mortality. ${ }^{2-4}$ On the other hands, hepatic steatosis may suggest subclinical metabolic risk factors such as cardiovascular changes ${ }^{5}$ and may accelerate the progression of the co-existing liver diseases. ${ }^{6}$

Hepatic steatosis is accumulation of triglycerides and other fats within the hepatocytes reflecting impairment of normal processes of synthesis and elimination of fat. The diagnosis of hepatic steatosis can be made when fat in the liver exceeds 5\% by weight. ${ }^{7}$ The current gold standard for evaluating steatsosis is liver biopsy, although its invasiveness limits its value as a screening tool especially in general population. ${ }^{8,9}$ Instead, the most practically used noninvasive means of detecting steatosis has been ultrasonography (US), although it can only detect steatosis of greater than $30 \% .{ }^{10}$ Recently, controlled attenuation parameter (CAP) being implemented on FibroScan ${ }^{\circledR}$ (Echosens, Paris, France) has been introduced in order to evaluate both steatosis and fibrosis simultaneously. ${ }^{11}$ It is a noninvasive means of quantitatively evaluating steatosis, and is reported to be highly sensitive in detecting low grade steatosis as fat deposition $>10 \%$ can be identified. ${ }^{12}$ In addition, CAP values may not be influenced by liver fibrosis and can be operated by a person without any US imaging skills without significant interoperator variability. ${ }^{13}$ Several recent studies reported that CAP is significantly correlated with the quantity of steatosis in patients with chronic liver diseases. ${ }^{12,14,15}$ However, not many studies investigated its role as a screening tool in general population. Although there may be debates as for the need of steatosis screening, a proportion of the population might benefit from the screening by identifying risk factors associated with metabolic derangements

Correspondence to: Jung Il Lee

Department of Internal Medicine, Gangnam Severance Hospital, Yonsei University College of Medicine, 211 Eonju-ro, Gangnam-gu, Seoul 135-720, Korea

Tel: +82-2-2019-3310, Fax: +82-2-3463-3882, E-mail: mdflorence@yuhs.ac

Received on May 29, 2014. Revised on July 15, 2014. Accepted on August 5, 2014. Published online on February 26,2015 pISSN 1976-2283 eISSN 2005-1212 http://dx.doi.org/10.5009/gnl14202

@ This is an Open Access article distributed under the terms of the Creative Commons Attribution Non-Commercial License (http://creativecommons.org/licenses/by-nc/3.0) which permits unrestricted non-commercial use, distribution, and reproduction in any medium, provided the original work is properly cited. 
such as diabetes mellitus and cardiovascular abnormalities. The aim of this study is to evaluate the usefulness of CAP in detecting steatosis among health checkup examinees and to assess its correlation with US.

\section{MATERIALS AND METHODS}

\section{Subjects}

From January 2013, 280 subjects who had CAP measured during health checkup program at Gangnam Severance Hospital, Seoul, Korea, were consecutively enrolled for this study. Other data including demographics, blood test results, liver stiffness measurement (LSM) and findings of abdominal US from database of Health Checkup Center were collected retrospectively. All subjects were examined between 8 and 11 AM after a 12-hour overnight fast. The study protocol was reviewed by Institutional Review Board of Gangnam Severance Hospital (number: 3-2014-0141). Conforming to the ethical guidelines of the 1975 Declaration of Helsinki, this study was approved by an ethics committee and granted an exemption.

\section{Blood samples}

Serological parameters included fasting glucose, aspartate aminotransferase (AST), alanine aminotransferase (ALT), $\gamma$-glutamyltranspeptidase, total bilirubin, platelet count, prothrombin time, total cholesterol, high-density lipoprotein (HDL) cholesterol, low-density lipoprotein (LDL) cholesterol and triglycerides. Blood test was performed on the same day of CAP measurement.

\section{Body composition}

Anthropometry and body composition were measured with an X-scan plus II (Jawon Medical Co., Ltd., Kyungsan, Korea). Anthropometric and body fat indices has been identified to be useful in showing body fat distribution and to have correlations with metabolic parameters. ${ }^{16-18}$ Weight $(\mathrm{kg})$ and height $(\mathrm{cm})$ were measured to the nearest $0.1 \mathrm{~kg}$ and $0.1 \mathrm{~cm}$, respectively. Bioelectrical impedance analysis was done for body composition via tetra-polar electrode method using eight touch electrodes. Mass of body fat, lean body mass, body mass index (BMI), percent body fat, waist to hip ratio, segmental analysis (lean body mass of arms, legs, and trunk), and basal metabolic rate were assessed.

\section{Ultrasonography}

Ultrasound scanning was performed by two professional radiologists using a 5-mHz transducer. Radiologists were not aware of the subjects' clinical details or laboratory findings. The criterion for fatty change of liver was hyperechogenic liver tissue with fine, tightly packed echoes. ${ }^{19}$ With the $5-\mathrm{mHz}$ transducer posterior beam penetration was an inevitable finding. The degree of fatty change was assessed by the fall in echo ampli- tude with depth (rate of posterior beam attenuation), increasing discrepancy of echo amplitude between liver and kidney, and loss of echoes from the walls of the portal veins. Mild fatty liver was defined by a slight increase in liver echogenicity, a slight exaggeration of liver and kidney echo discrepancy, and relative preservation of echoes from the walls of the portal vein. Moderate fatty liver was accompanied by loss of echoes from the walls of the portal veins, particularly from the peripheral branches, resulting in a featureless appearance of the liver. In addition, greater posterior beam attenuation was found and a greater discrepancy between hepatic and renal echoes. Severe fatty liver was recognized by a greater reduction in beam penetration, loss of echoes from most of the portal vein wall, including the main branches, and a large discrepancy between hepatic and renal echoes.

\section{CAP measurement and liver stiffness}

CAP was performed by a trained operator following the manufacturer's instruction. CAP assessment was on a Fibroscan $501^{\circledR}$ (Echosens, Paris, France) with the tip of M probe placing on the skin between the ribs over the right lobe of the liver. The CAP and liver stiffness were measured on the right lobe of the liver through the intercostal space. Ultrasound attenuation is only calculated when the LSM was valid in order to ensure an accurate attenuation value of the liver, and an attempt was made to collect $\geq 10$ valid LSMs. A success rate of $\geq 60 \%$ and the ratio of the interquartile range (IQR) of liver stiffness to the median (IQR/ $\left./ \mathrm{M}_{\mathrm{LSM}}\right) \leq 30 \%$ were considered reliable and used for the final analysis. As our working definition, steatosis grade was decided by cutoffs of CAP according to a previous report by Sasso et al. ${ }^{11} ; 238 \mathrm{~dB} / \mathrm{m}$ for $\mathrm{S} \geq 1,260 \mathrm{~dB} / \mathrm{m}$ for $\mathrm{S} \geq 2$, and 293 $\mathrm{dB} / \mathrm{m}$ for $\mathrm{S} 3$.

\section{Statistical analysis}

Continuous variables are expressed as mean \pm standard deviation. Categorical variables were expressed as counts and percent. Correlations among the variables were analyzed using Spearman correlation coefficient. The Student t-test was performed to compare variables between the groups. All statistical analyses were performed using the SPSS version 13.0 (SPSS Inc., Chicago, IL, USA). All statistical tests were two-sided and were evaluated at the 0.05 level of significance.

\section{RESULTS}

\section{Baseline characteristics}

The baseline characteristics of the subjects and the results of measured parameters are listed in Table 1 . The mean age was 51 years and $56.1 \%$ of the subjects were male. The mean values of CAP and LSM were $245.9 \pm 47.9 \mathrm{~dB} / \mathrm{m}$, and $4.6 \pm 2.8 \mathrm{kPa}$. The mean values of AST and ALT were $24.9 \pm 12.0$ and $27.4 \pm 25.0 \mathrm{IU} /$ L. The mean values of fasting glucose and total cholesterol were 
Table 1. Baseline Characteristics of Subjects $(n=280)$

\begin{tabular}{|c|c|}
\hline Characteristic & Value \\
\hline \multicolumn{2}{|l|}{ Clinical } \\
\hline Age, yr & $51 \pm 11$ \\
\hline Male sex & $157(56.1)$ \\
\hline DM & $18(6.7)$ \\
\hline Systolic BP, mm Hg & $124.29 \pm 14.67$ \\
\hline Diastolic BP, mm Hg & $77.7 \pm 9.75$ \\
\hline $\mathrm{CAP}, \mathrm{dB} / \mathrm{m}$ & $245.91 \pm 47.86$ \\
\hline Stiffness, kPa & $4.59 \pm 2.84$ \\
\hline HBsAg positive & $13(4.6)$ \\
\hline Anti-HCV positive & $1(0.4)$ \\
\hline \multicolumn{2}{|l|}{ Anthropometric } \\
\hline BMI, $\mathrm{kg} / \mathrm{m}^{2}$ & $23.90 \pm 3.03$ \\
\hline Body fat rate, $\%$ & $25.73 \pm 5.46$ \\
\hline Visceral fat grade, 1-24 & $10.19 \pm 3.37$ \\
\hline Visceral fat area, $\mathrm{cm}^{2}$ & $94.81 \pm 41.02$ \\
\hline Visceral fat, kg & $2.33 \pm 1.75$ \\
\hline Subcutaneous fat, kg & $14.54 \pm 3.80$ \\
\hline \multicolumn{2}{|l|}{ Biochemical } \\
\hline AST, IU/L & $24.91 \pm 12.02$ \\
\hline ALT, IU/L & $27.43 \pm 24.98$ \\
\hline GGT, IU/L & $37.80 \pm 56.71$ \\
\hline Fasting glucose, mg/dL & $100.89 \pm 15.97$ \\
\hline Total cholesterol, mg/dL & $200.26 \pm 37.68$ \\
\hline Triglyceride, $\mathrm{mg} / \mathrm{dL}$ & $121.15 \pm 72.69$ \\
\hline $\mathrm{HDL}, \mathrm{mg} / \mathrm{dL}$ & $51.34 \pm 12.90$ \\
\hline LDL, mg/dL & $121.69 \pm 31.65$ \\
\hline
\end{tabular}

Data are presented as mean \pm SD or number $(\%)$

$\mathrm{DM}$, diabetes mellitus; $\mathrm{BP}$, blood pressure; CAP, controlled attenuation parameter; $\mathrm{HBsAg}$, hepatitis B surface antigen; HCV, hepatitis $\mathrm{C}$ virus; BMI, body mass index; AST, aspartate aminotransferase; ALT, alanine aminotransferase; GGT, $\gamma$-glutamyltranspeptidase; HDL, highdensity lipoprotein; LDL, low-density lipoprotein.

$100.9 \pm 16.0 \mathrm{mg} / \mathrm{dL}$ and $200.3 \pm 37.7 \mathrm{mg} / \mathrm{dL}$. All the other mean values of blood chemistry were within normal ranges. Only a small number of subjects had diabetes (6.7\%), hepatitis B (4.6\%) or C $(0.4 \%)$ infection.

\section{Prevalence of steatosis}

US identified fatty liver in 119 subjects (42.5\%), while CAP measurement revealed S1-3 grade steatosis in 160 subjects (57.1\%). By US, 65 subjects (54.6\%) had mild fatty liver, 44 subjects (37.0\%) had moderate fatty liver, and 10 subjects (8.4\%) had severe fatty liver. By CAP value, subjects with S1, S2, and S3 grade steatosis were 59 (36.9\%), 58 (36.2\%) and 43 (26.9\%), respectively. The mean values of CAP in S0, S1, S2, and S3 groups were $203.3 \pm 28.4 \mathrm{~dB} / \mathrm{m}, 248.8 \pm 6.1 \mathrm{~dB} / \mathrm{m}, 274.3 \pm 8.5 \mathrm{~dB} /$ $\mathrm{m}$, and $322.4 \pm 22.2 \mathrm{~dB} / \mathrm{m}$, respectively (Table 2). Distribution of
Table 2. Fatty Change Noted by Ultrasonography and the Controlled Attenuation Parameter

\begin{tabular}{lcc}
\hline \multicolumn{1}{c}{ Characteristic } & No. $(\%)$ & Mean \pm SD, dB/m \\
\hline Fatty change by US $(\mathrm{n}=119)$ & & \\
$\quad$ Mild fatty liver & $65(54.6)$ & - \\
Moderate fatty liver & $44(37.0)$ & - \\
Severe fatty liver & $10(8.4)$ & - \\
Fatty change by CAP $(\mathrm{n}=160), \mathrm{dB} / \mathrm{m}$ & & \\
S1 $(238$ to $<260)$ & $59(36.9)$ & $248.83 \pm 6.14$ \\
S2 $(260$ to $<293)$ & $58(36.2)$ & $274.33 \pm 8.53$ \\
S3 $(\geq 293)$ & $43(26.9)$ & $322.35 \pm 22.20$ \\
\hline
\end{tabular}

US, ultrasonography; CAP, controlled attenuation parameter.

Table 3. Correlation between the Controlled Attenuation Parameter and Clinical Parameters

\begin{tabular}{lcc}
\hline \multicolumn{1}{c}{ Parameter } & Correlation $(\mathrm{r})$ & $\mathrm{p}$-value \\
\hline US finding (fatty liver grade) & 0.472 & $<0.001$ \\
BMI & 0.445 & $<0.001$ \\
Visceral fat grade & 0.421 & $<0.001$ \\
Systolic BP & 0.223 & $<0.001$ \\
Fasting glucose & 0.229 & $<0.001$ \\
Triglyceride & 0.269 & $<0.001$ \\
HDL & -0.155 & 0.012 \\
LDL & 0.182 & 0.003 \\
\hline
\end{tabular}

US, ultrasonography; BMI, body mass index; BP, blood pressure; HDL, high-density lipoprotein; LDL, low-density lipoprotein.

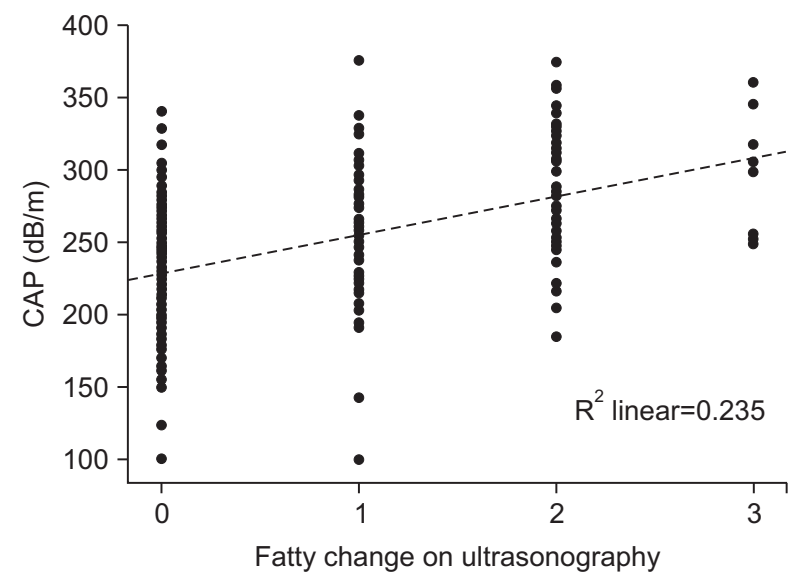

Fig. 1. Scatterplot and regression line showing a positive correlation between the grade of fatty change ( 0 , normal; 1 , mild; 2 , moderate; and 3 , severe) detected by ultrasonography and the controlled attenuation parameter in the checkup subjects.

CAP was plotted by the degree of fatty change in US (Fig. 1).

\section{Correlations with CAP}

CAP was significantly correlated with parameters as shown in Table 3. BMI, systolic blood pressure (BP), fasting glucose, 
triglyceride, LDL, and grade of fatty liver by US showed significant positive correlations with CAP value. HDL level showed a negative significant correlation with CAP value.

\section{Characteristics of subjects whose steatosis was detected only by CAP value}

Among 161 subjects whose liver echogenicity was normal in US, steatosis was detected in 65 subjects $(65 / 161,40.4 \%)$ by CAP value, and most of them (53.8\%) had S1 grade CAP value. None of patients whose liver steatosis was detected by US had normal values on CAP evaluation. We compared clinical parameters between US-CAP concordant subjects (normal liver echogenicity in US and So grade of CAP value) and US-CAP discordant subjects (normal liver echogenicity in US but steatosis by CAP value). Subjects whose steatosis was recognized by CAP had significantly higher BMI, body fat rate, visceral fat grade, systolic BP, triglyceride, LDL, and low HDL than subjects without steatosis that was confirmed by the both tools, US and CAP measurements (Table 4). However, mean values of diastolic $\mathrm{BP}$, fasting glucose, total cholesterol, AST, and ALT were not different statistically.

\section{DISCUSSION}

In our study, liver steatosis, evaluated by CAP was well correlated with clinical parameters that were associated with metabolic derangements. In addition, CAP measurement was useful in identifying the low grade steatosis that was not conspicuous on US, and thus might be useful in early detection of metabolic derangements in health checkup examinees.

The need for steatosis screening in general population is still under controversy. However, health checkup is often provided for persons that do not possess overt, serious health problems in order to prevent disease by recognizing subtle signs jeopardizing healthy condition, and several studies suggest hepatic steatosis as an early predictor of cardiovascular and metabolic derangements. ${ }^{20,21}$ NAFLD has been suggested as an indicator of preclinical insulin resistance even in nonobese individuals, and some investigators even recommended young NAFLD patients to undergo oral glucose tolerance test in order to predict the risk of type 2 diabetes. ${ }^{21-23}$ Furthermore, NAFLD is reported to suggest subclinical cardiovascular changes such as increased arterial stiffness and carotid arterial wall thickness. ${ }^{5,24-26}$ Therefore, early detection of NAFLD might contribute in predicting and preventing serious and prevalent metabolic diseases such as type 2 diabetes or cardiovascular disease. In accordance with these findings, our results also showed that the presence of hepatic steatosis by CAP correlated with variables suggestive of poor metabolic condition. Fortunately, simple hepatic steatosis without inflammation and fibrosis is a reversible condition which can be normalized after lifestyle modification including diet and/or physical activity intervention. ${ }^{27,28}$ It would be interesting to investigate whether alleviated hepatic steatosis would reduce the metabolic risks, and such studies would be able to determine the usefulness of steatosis screening.

Currently, US is recognized as the most useful and widely accepted noninvasive technique for the detection of liver steatosis. However, US has its pitfalls as its results tend to be operator dependent and its grading system is somewhat subjective. ${ }^{29}$ In addition, quantification of fatty change is limited as it is described in only four categories: normal, mild, moderate, and severe. Some investigators even report relatively low sensitivity of US in identifying existence of fatty liver. ${ }^{30,31}$ Therefore, in comparison with the histological studies, some review articles raise questions on the diagnostic ability and reliability of US in detecting fatty liver. ${ }^{32,33}$ On the other hands, CAP measures and

Table 4. Clinical Implication of Fatty Change Not Detected by Ultrasonography but Detected by the Controlled Attenuation Parameter

\begin{tabular}{lccc}
\hline \multicolumn{1}{c}{ Parameter } & Normal liver by US and CAP $(\mathrm{n}=96)$ & Fatty liver not by US but by CAP $(\mathrm{n}=65)$ & $\mathrm{p}$-value \\
\hline BMI, $\mathrm{kg} / \mathrm{m}^{2}$ & $22.03 \pm 2.61$ & $23.73 \pm 1.45$ & $<0.001$ \\
Body fat rate, \% & $24.21 \pm 5.37$ & $26.24 \pm 5.64$ & 0.022 \\
Visceral fat grade & $7.88 \pm 3.43$ & $10.51 \pm 2.55$ & $<0.001$ \\
Systolic BP, mm Hg & $119.5 \pm 12.79$ & $124.42 \pm 15.85$ & 0.032 \\
Diastolic BP, mm Hg & $74.51 \pm 8.79$ & $76.63 \pm 9.95$ & 0.156 \\
Fasting glucose, mg/dL & $95.99 \pm 10.85$ & $95.85 \pm 12.22$ & 0.641 \\
Total cholesterol, mg/dL & $192.46 \pm 35.83$ & $200.63 \pm 37.39$ & 0.165 \\
AST, IU/L & $23.78 \pm 13.87$ & $21.37 \pm 5.78$ & 0.187 \\
ALT, IU/L & $22.71 \pm 30.34$ & $20.60 \pm 10.46$ & 0.131 \\
Triglyceride, mg/dL & $89.17 \pm 44.15$ & $106.48 \pm 49.66$ & 0.022 \\
HDL, $\mathrm{mg} / \mathrm{dL}$ & $55.31 \pm 12.42$ & $50.92 \pm 10.54$ & 0.021 \\
LDL, $\mathrm{mg} / \mathrm{dL}$ & $114.95 \pm 33.17$ & $126.02 \pm 28.63$ & 0.030 \\
\hline
\end{tabular}

US, ultrasonography; CAP, controlled attenuation parameter; BMI, body mass index; BP, blood pressure; AST, aspartate aminotransferase; ALT, alanine aminotransferase; HDL, high-density lipoprotein; LDL, low-density lipoprotein. 
describes hepatic steatosis in a numeric scale, which may produce more objective results. The numeric scales are often useful when the examinees want to compare their hepatic fatty values over time after serial measurements. CAP seems to be able to produce objective assessment of hepatic steatosis, along with noninvasively measured liver fibrosis, and it is operator independent as well as easily accessible. CAP may be a noninvasive and practical means of detecting hepatic steatosis in patients as well as in general population. However, until now, there is no consensus on cutoff values regarding steatosis grade. In addition, there are no validated indices that describe the reliability of CAP values. For liver stiffness, IQR/M is known to be a factor of reliability. However, in a previous report comparing histology and $\mathrm{CAP}, \mathrm{IQR} / \mathrm{M}$ of CAP was not an independent predictor of discordance between steatosis grade and CAP. ${ }^{34}$ Further study is warranted to decide optimal cutoff value for detecting early fatty changes and to identify means of assessing the reliability of the CAP study.

Our study reports the correlation coefficient of 0.472 between findings of US and those of the CAP study and it should be pointed out that the level of correlation is not so strong. Unfortunately reports on the correlation between results from US and that of CAP are lacking, and more studies on this matter are needed as indicated in a recent review article. ${ }^{35}$

This study has several limitations. First, comparison with the histologic grade was not included. To be conducted with data from health checkup, invasive confirmation with biopsy was unrealistic and even unethical. Second, cause of hepatic steatosis was not clearly depicted. As these are real life data and almost every man has social drinking, alcoholic or nonalcoholic causes could not be easily discriminated. Third, we could not show the relation between CAP and insulin resistance since serum insulin level was not included in health checkup item.

In conclusion, among health checkup examinees, CAP seems to be useful in detecting hepatic steatosis to predict metabolic derangement. Even with normal US findings, those subjects whose hepatic steatosis could be diagnosed by CAP had worse metabolic parameters compared with those that were free of fatty liver after both US and CAP studies. Histologic validation is warranted to set cutoffs of CAP for steatosis grade in subjects without other definite causes of the chronic liver diseases.

\section{CONFLICTS OF INTEREST}

No potential conflict of interest relevant to this article was reported.

\section{ACKNOWLEDGEMENTS}

This study was supported by a grant of the Korea Healthcare Technology R\&D Project, Ministry of Health and Welfare, Republic of Korea (number: HI10C2020).

\section{REFERENCES}

1. Haque M, Sanyal AJ. The metabolic abnormalities associated with non-alcoholic fatty liver disease. Best Pract Res Clin Gastroenterol 2002;16:709-731.

2. Harrison SA, Torgerson S, Hayashi PH. The natural history of nonalcoholic fatty liver disease: a clinical histopathological study. Am J Gastroenterol 2003;98:2042-2047.

3. Fassio E, Alvarez E, Domínguez N, Landeira G, Longo C. Natural history of nonalcoholic steatohepatitis: a longitudinal study of repeat liver biopsies. Hepatology 2004;40:820-826.

4. Adams LA, Sanderson S, Lindor KD, Angulo P. The histological course of nonalcoholic fatty liver disease: a longitudinal study of 103 patients with sequential liver biopsies. J Hepatol 2005;42:132138

5. Kim NH, Park J, Kim SH, et al. Non-alcoholic fatty liver disease, metabolic syndrome and subclinical cardiovascular changes in the general population. Heart 2014;100:938-943.

6. Powell EE, Jonsson JR, Clouston AD. Metabolic factors and nonalcoholic fatty liver disease as co-factors in other liver diseases. Dig Dis 2010;28:186-191.

7. Hübscher SG. Histological assessment of non-alcoholic fatty liver disease. Histopathology 2006;49:450-465.

8. Rockey DC, Caldwell SH, Goodman ZD, Nelson RC, Smith AD; American Association for the Study of Liver Diseases. Liver biopsy. Hepatology 2009;49:1017-1044.

9. Myers RP, Fong A, Shaheen AA. Utilization rates, complications and costs of percutaneous liver biopsy: a population-based study including 4275 biopsies. Liver Int 2008;28:705-712.

10. Dasarathy S, Dasarathy J, Khiyami A, Joseph R, Lopez R, McCullough AJ. Validity of real time ultrasound in the diagnosis of hepatic steatosis: a prospective study. J Hepatol 2009;51:10611067.

11. Sasso M, Beaugrand M, de Ledinghen V, et al. Controlled attenuation parameter (CAP): a novel VCTE(TM) guided ultrasonic attenuation measurement for the evaluation of hepatic steatosis: preliminary study and validation in a cohort of patients with chronic liver disease from various causes. Ultrasound Med Biol 2010;36: 1825-1835.

12. de Lédinghen V, Vergniol J, Foucher J, Merrouche W, le Bail B. Non-invasive diagnosis of liver steatosis using controlled attenuation parameter (CAP) and transient elastography. Liver Int 2012; 32:911-918.

13. Ratziu V, Bellentani S, Cortez-Pinto H, Day C, Marchesini G. A position statement on NAFLD/NASH based on the EASL 2009 special conference. J Hepatol 2010;53:372-384.

14. Sasso M, Tengher-Barna I, Ziol M, et al. Novel controlled attenuation parameter for noninvasive assessment of steatosis using Fibroscan $\left({ }^{\circledR}\right)$ : validation in chronic hepatitis C. J Viral Hepat 2012; 19:244-253.

15. Myers RP, Pollett A, Kirsch R, et al. Controlled Attenuation Parameter (CAP): a noninvasive method for the detection of hepatic 
steatosis based on transient elastography. Liver Int 2012;32:902910.

16. De Lorenzo A, Di Campli C, Andreoli A, Sasso GF, Bonamico M, Gasbarrini A. Assessment of body composition by bioelectrical impedance in adolescent patients with celiac disease. Am J Gastroenterol 1999;94:2951-2955.

17. Nagaya T, Yoshida H, Takahashi H, Matsuda Y, Kawai M. Body mass index (weight/height2) or percentage body fat by bioelectrical impedance analysis: which variable better reflects serum lipid profile? Int J Obes Relat Metab Disord 1999;23:771-774.

18. Kobayashi J, Murano S, Kawamura I, et al. The relationship of percent body fat by bioelectrical impedance analysis with blood pressure, and glucose and lipid parameters. J Atheroscler Thromb 2006;13:221-226.

19. Foster KJ, Dewbury KC, Griffith AH, Wright R. The accuracy of ultrasound in the detection of fatty infiltration of the liver. Br J Radiol 1980;53:440-442.

20. Kim HJ, Kim HJ, Lee KE, et al. Metabolic significance of nonalcoholic fatty liver disease in nonobese, nondiabetic adults. Arch Intern Med 2004;164:2169-2175.

21. Chon CW, Kim BS, Cho YK, et al. Effect of nonalcoholic Fatty liver disease on the development of type 2 diabetes in nonobese, nondiabetic Korean men. Gut Liver 2012;6:368-373.

22. Yun JW, Cho YK, Park JH, et al. Abnormal glucose tolerance in young male patients with nonalcoholic fatty liver disease. Liver Int 2009;29:525-529.

23. Choi JH, Rhee EJ, Bae JC, et al. Increased risk of type 2 diabetes in subjects with both elevated liver enzymes and ultrasonographically diagnosed nonalcoholic fatty liver disease: a 4-year longitudinal study. Arch Med Res 2013;44:115-120.

24. Kim HC, Kim DJ, Huh KB. Association between nonalcoholic fatty liver disease and carotid intima-media thickness according to the presence of metabolic syndrome. Atherosclerosis 2009;204:521525.
25. Kim BJ, Kim NH, Kim BS, Kang JH. The association between nonalcoholic fatty liver disease, metabolic syndrome and arterial stiffness in nondiabetic, nonhypertensive individuals. Cardiology 2012;123:54-61.

26. Thakur ML, Sharma S, Kumar A, et al. Nonalcoholic fatty liver disease is associated with subclinical atherosclerosis independent of obesity and metabolic syndrome in Asian Indians. Atherosclerosis 2012;223:507-511.

27. Sullivan S, Kirk EP, Mittendorfer B, Patterson BW, Klein S. Randomized trial of exercise effect on intrahepatic triglyceride content and lipid kinetics in nonalcoholic fatty liver disease. Hepatology 2012;55:1738-1745.

28. Wong VW, Chan RS, Wong GL, et al. Community-based lifestyle modification programme for non-alcoholic fatty liver disease: a randomized controlled trial. J Hepatol 2013;59:536-542.

29. Hernaez R, Lazo M, Bonekamp S, et al. Diagnostic accuracy and reliability of ultrasonography for the detection of fatty liver: a meta-analysis. Hepatology 2011;54:1082-1090.

30. Cardi M, Muttillo IA, Amadori L, et al. Superiority of laparoscopy compared to ultrasonography in diagnosis of widespread liver diseases. Dig Dis Sci 1997;42:546-548.

31. Hepburn MJ, Vos JA, Fillman EP, Lawitz EJ. The accuracy of the report of hepatic steatosis on ultrasonography in patients infected with hepatitis $\mathrm{C}$ in a clinical setting: a retrospective observational study. BMC Gastroenterol 2005;5:14.

32. Wieckowska A, Feldstein AE. Diagnosis of nonalcoholic fatty liver disease: invasive versus noninvasive. Semin Liver Dis 2008;28:386-395.

33. Adams LA, Talwalkar JA. Diagnostic evaluation of nonalcoholic fatty liver disease. J Clin Gastroenterol 2006;40 Suppl 1:S34-S38.

34. Rocha LA, Aleixo A, Allen G, et al. Specimen collection: an essential tool. Science 2014;344:814-815.

35. Castera L, Vilgrain V, Angulo P. Noninvasive evaluation of NAFLD. Nat Rev Gastroenterol Hepatol 2013;10:666-675. 